\title{
Neurobiological Approach of Catatonia and Treatment Perspectives
}

\author{
Pierre Ellul and Walid Choucha* \\ Department of Psychiatry, Assistance Publique-Hôpitaux de Paris, Pitié-Salpétrière University Hospital, University Pierre et \\ Marie Curie, Paris, France \\ Keywords: catatonia, emotion regulation, prefrontal cortex, GABA-A receptors, clinical neurosciences \\ psychopharmacology
}

\section{INTRODUCTION}

Catatonia was described for the first time by Kahlbaum in 1874 (1). It can be defined schematically as a motor dysregulation syndrome accompanied with a behavioral component. There are three main forms of catatonia: (i) akinetic, (ii) hyperkinetic, and (iii) malignant catatonia (2). These various phenotypes of the same syndrome led to a clinical heterogeneousness making the catatonia difficult to recognize and diagnose. It seems, however, that certain clinical signs occur with a greater frequency in catatonia. Indeed, in a study involving more than 230 catatonic patients, the "staring," was found in more than $80 \%$ of cases. Among other frequent signs, were the immobility in $70 \%$ of cases, the mutism in $60 \%$ of patients, and the withdrawal in $50 \%$ of them (3). Various specific scales were developed to allow a more accurate diagnostic approach to catatonia. The Bush-Francis catatonia rating scale is the most commonly used one. It has many advantages: in addition to having a sensitivity of reaching $100 \%$ and a specificity between 75 and $100 \%$, it is fast and easy to use in daily clinical practice (4). It is important to note that catatonia is a transnosographic syndrome with various underlying psychiatric and somatic causes. Most common somatic causes include epilepsy, systemic lupus erythematosus, intermittent porphyria, traumatic brain injury, dementia, encephalopathies (autoimmune, paraneoplastic, Hashimoto, etc.) (5). Furthermore, catatonia is found among $10 \%$ of psychiatric inpatients (6). Under the influence of Kraeplin, catatonia was linked for a long time exclusively with schizophrenia (7). However, recent epidemiological studies showed that schizophrenia is found only in $20 \%$ of catatonic cases while mood disorders underlie $45 \%$ of cases (8). Catatonia is also frequent in children and adolescents, particularly in autism spectrum disorders where the prevalence varies between 12 and 17\% (9). Despite these clinical and epidemiological facts, few data exist concerning the exact pathophysiological mechanisms underlying this syndrome. In this paper, we will make the synthesis of the existing data concerning the neurocognitive and neurobiological mechanisms involved in the akinetic forms of catatonia.

\section{PREFRONTAL PHYSIOLOGY OF EMOTIONS}

Accepted: 11 December 2015 Published: 24 December 2015

Citation: Ellul P and Choucha W (2015)

Neurobiological Approach of Catatonia and Treatment Perspectives.

Front. Psychiatry 6:182. doi: 10.3389/fpsyt.2015.00182

Moskowitz considered catatonia as an evolutionary remainder of defense strategies associated with intense fear (10). It seems that in front of predators, several survival behaviors have been developed. Among them, the most known one was the "fight or flight" strategy. In cases where none of these two options was possible, a third strategy called "tonic immobility" (TI) would be set up which consists of a tonic suspension of motor activity. This defense strategy is based on the fact that many predators are attracted by their prey's movements. This hypothesis seems to be confirmed by the subjective experience of catatonic patients. Indeed, once remitted from their catatonia, patients 
report having felt invaded by a major and uncontrollable anxiety. Conversely, they do not seem to have been aware of their motor state (11). To better understand the brain abnormalities found in catatonic patients, it seems essential to focus on the neurological mechanisms involved in the physiological integration of emotions. The amygdala appears to have a central role in emotional regulation processes, particularly negative emotions, such as fear or anxiety (12). The environmental informations are conveyed to different brain areas according to each specific sensory modality (for example, visual stimuli are conveyed to occipital cortex, auditory informations to temporal cortex, etc.) and secondarily sent to the amygdala, serving as an emotional crossroad (12). To start making the link amygdala/emotions/catatonia, it may already be interesting to note that, in animals, hyperactivation of the amygdala is responsible of a freezing behavior, which is similar to TI and symptoms found in the akinetic forms of catatonia (13). Once informations are integrated in the amygdala, they may, depending on the emotional valence, activate different neural circuits. In particular, functional MRI studies found that negative emotions are associated with increased activation of the orbitofrontal cortex (OFC) and the ventromedial prefrontal cortex (VMPFC) and decreased activity of dorsolateral prefrontal cortex (DLPFC). The exact opposite activation profile occurs with positive emotions making the PFC a regulating crossroad depending on the emotion type (14). These variations in the activation/deactivation pattern seem to be modulated by the GABAergic system (15). Each of the involved brain areas is associated with specific functions. OFC is involved in decoding the emotional environmental situations and in taking decisions depending on the context (16). VMPFC is considered as a self-centered emotional integration center. It will allow, in some way, perceiving emotions (17). Regarding DLPFC role, it is implicated in cognitive processes and action planification (18). It will allow a cognitive approach in understanding emotions and a negative feedback on emotional processes, especially amygdalian ones. In other words, DLPFC performs cognitive control of emotions $(19,20)$. Furthermore, the DLPFC is a major integrative crossroad. Indeed, it receives informations, among others, from the posterior parietal cortex, which is itself involved in negative emotions (21). DLPFC will then project mainly on motor areas (22). It is therefore considered as a sensorimotor associative region bridging the gap between emotional cognitive perception and motor skills (23).

\section{PATHOPHYSIOLOGY OF CATATONIA}

To confirm TI hypothesis in catatonia, functional MRI studies have investigated catatonic brain activation during emotional processing. One of them compared patients who remitted from catatonia to non-catatonic psychiatric patients and finally to healthy subjects. Authors found among remitted catatonic patients, a hyperactivation of the OFC and the VMPFCs during negative emotions compared to the two other groups (24). Furthermore, statistical analysis showed a positive correlation between the hyperactivation of the OFC and behavioral/emotional symptoms and between the hyperactivation of VMPFCs and motor symptoms (24). The authors also found alterations in corticocortical connections between (i) OFC and VMPFC; (ii) between VMPFC/DLPFC and motor/premotor cortex (24). Another study examined the effect of lorazepam [a benzodiazepine known for its effectiveness in catatonia $(25,26)]$ on the modulation of activation patterns of the PFC during negative emotions. A decrease in OFC and VMPC hyperactivation was observed with lorazepam in successfully treated catatonic patients, leading to a regularization of the OCF activity, compared to control (27). It seems that GABA and especially the GABA-A receptors may play an important role in the pathophysiology of catatonia. One study looked at the density of GABA-A receptors as well as changes in cerebral perfusion in catatonic patients compared to non-catatonic psychiatric patients and healthy subjects. The authors found a decrease of the GABA-A receptors density in the DLPFC associated with a decrease of cerebral perfusion in prefrontal and posterior parietal cortex (28). Moreover, motor and affective symptoms were significantly associated with the decreased GABA-A receptors density in the DLPFC (28). Involvement of the DLPF in catatonia has also been demonstrated by indirect evidences, such as the therapeutic efficacy of high-frequency transcranial magnetic stimulation applied to this area $(29,30)$. Some authors tried to correlate brain activation changes with different catatonic symptoms, especially motor ones. A controlled study using different motor tasks (idle status, self-initiated movements, and movements on request) showed a decreased activity of the prefrontal cortex, the parietal cortex, and the supplementary motor area in catatonic patients compared to controls (31). These changes persisted even after remission. Specifically, it seems that it is the latency of late motors potentials at the frontoparietal line that is affected in catatonia with GABAergic altered sensitivity compared to control (31). These results are in agreement with the fact that catatonic patients may successfully initiate movements but present difficulties in terminating them $(32,33)$. Another study examined cerebral perfusion changes in catatonic patients before and after treatment with electroconvulsive therapy (ECT) and found increased perfusion in the parietal cortex after successful treatment (34). Indeed, it appears that the parietal cortex may play an important role in motricity as demonstrated by the occurrence of a catatonic state in patients with a parietal lesion (35). Considering these studies, it seems that different brain areas, in addition to the PFC, are involved in the catatonia. Neurocognitive studies showed a selective deficit in visual-spatial performances in catatonic patients compared to controls (36). These results confirmed indirectly the role of the posterior parietal cortex dysfunction in catatonia as it is broadly implicated in visual-spatial performance (37, 38). A positive correlation was also found between the activity of mirror neurons and echophenomena (echopraxia, echolalia) and their disappearance after administration of lorazepam (39). Indeed, these echo-phenomena seem to be attributed to the disinhibition of the mirror neurons, which would be related to a control deficit of the GABAergic system, within the OCF, the VMPFC, the DLPFC, and the parietal cortex (40). Moreover, glutamate seems to be involved in catatonia as well, particularly via the NMDA receptors activity. These assumptions are based primarily on the efficacy of NMDA-receptor antagonists, such as amantadine in catatonia and also in cases of catatonia related to anti-NMDA receptor encephalitis (41-44). Amantadine may act 
by decreasing cerebral glutamatergic activity creating a relative increase in the inhibitory GABAergic activity (45).

\section{CONCLUSION AND PERSPECTIVE}

The exact mechanisms underlying the pathophysiology of catatonia still remain a mystery. It seems that some people are more predisposed than others to develop this syndrome. Indeed, most studies agreed on the existence of trait markers, especially GABAergic cortical dysregulation, resulting in the failure of cognitive control of emotions. When intense emotional changes generated by psychiatric disorders (depression, mania, and schizophrenia) are added to these predispositions, this would precipitate a state of TI: in other words, catatonia. Schematically, in response to negative emotions, the GABAergic inhibitory control at the OFC could not take place, leading to a deregulation in VMPFC/DLPFC balance, which would then prevent cognitive control of negative emotions by the DLPFC. In addition, the deficit in DLPFC activation would impair its associative function, and particularly its connectivity with the parietal cortex and the motor areas leading to the occurrence of the motor signs found in akinetic forms of catatonia. There are many limits to the studies mentioned above: (i) they included a small number of patients, (ii) few of them compared catatonic patients to healthy controls or to controls with psychiatric disorders, and (iii) clinical heterogeneity of catatonia was not taken in consideration in these studies. In the future, it might be interesting to develop clinico-morphological correlation studies

\section{REFERENCES}

1. Fink M. Rediscovering catatonia: the biography of a treatable syndrome. Acta Psychiatr Scand Suppl (2013) 127(441):1-47. doi:10.1111/acps.12038

2. Francis A. Catatonia: diagnosis, classification, and treatment. Curr Psychiatry Rep (2010) 12(3):180-5. doi:10.1007/s11920-010-0113-y

3. Wilson JE, Niu K, Nicolson SE, Levine SZ, Heckers S. The diagnostic criteria and structure of catatonia. Schizophr Res (2015) 164:256-62. doi:10.1016/j. schres.2014.12.036

4. Sienaert P, Rooseleer J, De Fruyt J. Measuring catatonia: a systematic review of rating scales. J Affect Disord (2011) 135(1-3):1-9. doi:10.1016/j. jad.2011.02.012

5. Smith JH, Smith VD, Philbrick KL, Kumar N. Catatonic disorder due to a general medical or psychiatric condition. J Neuropsychiatry Clin Neurosci (2012) 24(2):198-207. doi:10.1176/appi.neuropsych.11060120

6. Francis A, Fink M, Appiani F, Bertelsen A, Bolwig TG, Bräunig P, et al. Catatonia in diagnostic and statistical manual of mental disorders, fifth edition. J ECT (2010) 26(4):246-7. doi:10.1097/YCT.0b013e3181fe28bd

7. Fink M, Shorter E, Taylor MA. Catatonia is not schizophrenia: Kraepelin's error and the need to recognize catatonia as an independent syndrome in medical nomenclature. Schizophr Bull (2010) 36(2):314-20. doi:10.1093/schbul/sbp059

8. Rosebush PI, Mazurek MF. Catatonia and its treatment. Schizophr Bull (2010) 36(2):239-42. doi:10.1093/schbul/sbp141

9. Wing L, Shah A. Catatonia in autistic spectrum disorders. Br J Psychiatry (2000) 176(4):357-62. doi:10.1192/bjp.176.4.357

10. Moskowitz AK. "Scared stiff”: catatonia as an evolutionary-based fear response. Psychol Rev (2004) 111(4):984-1002. doi:10.1037/0033-295X.111.4.984

11. Northoff G, Krill W, Wenke J, Gille B, Russ M, Eckert J, et al. Major differences in subjective experience of akinetic states in catatonic and parkinsonian patients. Cogn Neuropsychiatry (1998) 3(3):161-78.

12. Janak PH, Tye KM. From circuits to behaviour in the amygdala. Nature (2015) 517(7534):2842-9. doi:10.1038/nature14188 with particular attention to the potential role of the amygdala in catatonia. This approach might open the way for new therapeutic options targeting the amygdala. For example, oxytocin seems to have a direct attenuating effect on reactions of fear and anxiety by acting directly on the amygdala (46). Other studies focusing on the role of glutamate in catatonia could pave the way for therapeutic innovations. For example, it is possible to imagine the use of drugs with dual action on both GABAergic and glutamatergic systems to treat resistant forms of catatonia. Some drugs having such properties are already available, especially acamprosate and lamotrigine which possesses this dual receptor profile (47). Rapid and accurate diagnosis and treatment of catatonia is crucial in clinical practice not only to avoid somatic complications but to avoid the development of resistance to treatment as well. Indeed, the longer catatonic symptoms last, the more will be the risk of developing resistance to treatment (48). Consequently, rapid achievement of full remission of catatonic symptoms should be an essential goal.

\section{AUTHOR CONTRIBUTIONS}

$\mathrm{PE}$ and WC participated both to research and writing of the paper in the same way.

\section{ACKNOWLEDGMENTS}

$\mathrm{PE}$ and WC report no financial relationships with commercial interests.

13. Arruda-Carvalho M, Clem RL. Pathway-selective adjustment of prefrontal-amygdala transmission during fear encoding. J Neurosci (2014) 34(47):15601-9. doi:10.1523/JNEUROSCI.2664-14.2014

14. Phan KL, Wager T, Taylor SF, Liberzon I. Functional neuroanatomy of emotion: a meta-analysis of emotion activation studies in PET and fMRI. Neuroimage (2002) 16(2):331-48. doi:10.1006/nimg.2002.1087

15. Northoff G, Witze T, Richter A, Gessner M, Schlagenhauf F, Fell J, et al. GABAergic modulation of prefrontal spatio-temporal activation pattern during emotional processing: a combined fMRI/MEG study with placebo and lorazepam. J Cogn Neurosci (2002) 14(3):348-70. doi:10.1162/089892902317361895

16. Rolls ET. Précis of the brain and emotion. Behav Brain Sci (2000) 23(2):177-91; discussion 192-233.

17. Kuo M-F, Nitsche MA. Exploring prefrontal cortex functions in healthy humans by transcranial electrical stimulation. Neurosci Bull (2015) 31(2):198206. doi:10.1007/s12264-014-1501-9

18. Tanji J, Hoshi E. Behavioral planning in the prefrontal cortex. Curr Opin Neurobiol (2001) 11(2):164-70.

19. Mitchell RLC, Phillips LH. The overlapping relationship between emotion perception and theory of mind. Neuropsychologia (2015) 70:1-10. doi:10.1016/j. neuropsychologia.2015.02.018

20. Morawetz C, Bode S, Baudewig J, Kirilina E, Heekeren HR. Changes in effective connectivity between dorsal and ventral prefrontal regions moderate emotion regulation. Cereb Cortex (2015). doi:10.1093/cercor/ bhv005

21. Hallam GP, Webb TL, Sheeran P, Miles E, Wilkinson ID, Hunter MD, et al. The neural correlates of emotion regulation by implementation intentions. PLoS One (2015) 10(3):e0119500. doi:10.1371/journal.pone.0119500

22. Goldman-Rakic PS, Bates JF, Chafee MV. The prefrontal cortex and internally generated motor acts. Curr Opin Neurobiol (1992) 2(6):830-5.

23. Kehrer S, Kraft A, Koch SP, Kathmann N, Irlbacher K, Brandt SA. Timing of spatial priming within the fronto-parietal attention network: a TMS study. Neuropsychologia (2015) 74:30-6. doi:10.1016/j.neuropsychologia.2014.11.017 
24. Northoff G, Kötter R, Baumgart F, Danos P, Boeker H, Kaulisch T, et al. Orbitofrontal cortical dysfunction in akinetic catatonia: a functional magnetic resonance imaging study during negative emotional stimulation. Schizophr Bull (2004) 30(2):405-27.

25. Sharma CM, Jena S, Sharma D, Agrawal RP. Role of lorazepam challenge test in childhood catatonia. J Pediatr Neurosci (2014) 9(3):301-3. doi:10.4103/1817-1745.147611

26. Lin C-C, Huang T-L. Lorazepam-diazepam protocol for catatonia in schizophrenia: a 21-case analysis. Compr Psychiatry (2013) 54(8):1210-4. doi:10.1016/j.comppsych.2013.06.003

27. Richter A, Grimm S, Northoff G. Lorazepam modulates orbitofrontal signal changes during emotional processing in catatonia. Hum Psychopharmacol (2010) 25(1):55-62. doi:10.1002/hup.1084

28. Northoff G, Steinke R, Czcervenka C, Krause R, Ulrich S, Danos P, et al. Decreased density of GABA-A receptors in the left sensorimotor cortex in akinetic catatonia: investigation of in vivo benzodiazepine receptor binding. J Neurol Neurosurg Psychiatry (1999) 67(4):445-50.

29. Trojak B, Meille V, Bonin B, Chauvet-Geliner J-C. Repetitive transcranial magnetic stimulation for the treatment of catatonia: an alternative treatment to electroconvulsive therapy? J Neuropsychiatry Clin Neurosci (2014) 26(2):E42-3. doi:10.1176/appi.neuropsych.13050102

30. Cristancho MA, Cristancho P, O’Reardon JP. Other therapeutic psychiatric uses of superficial brain stimulation. Handb Clin Neurol (2013) 116:415-22. doi:10.1016/B978-0-444-53497-2.00034-6

31. Northoff G, Pfennig A, Krug M, Danos P, Leschinger A, Schwarz A, et al. Delayed onset of late movement-related cortical potentials and abnormal response to lorazepam in catatonia. Schizophr Res (2000) 44(3):193-211.

32. Northoff G, Wenke J, Krill W, Pflug B. Ball experiments in 32 acute akinetic catatonic patients: deficits of internal initiation and generation of movements. Mov Disord (1995) 10(5):589-95.

33. Northoff G. What catatonia can tell us about "top-down modulation": a neuropsychiatric hypothesis. Behav Brain Sci (2002) 25(5):555-77; discussion 578-604.

34. Escobar R, Rios A, Montoya ID, Lopera F, Ramos D, Carvajal C, et al. Clinical and cerebral blood flow changes in catatonic patients treated with ECT. J Psychosom Res (2000) 49(6):423-9.

35. Fukutake T, Hirayama K, Komatsu T. Transient unilateral catalepsy and right parietal damage. Jpn J Psychiatry Neurol (1993) 47(3):647-50.

36. Northoff G, Nagel D, Danos P, Leschinger A, Lerche J, Bogerts B. Impairment in visual-spatial function in catatonia: a neuropsychological investigation. Schizophr Res (1999) 37(2):133-47.

37. Swaminathan SK, Freedman DJ. Preferential encoding of visual categories in parietal cortex compared with prefrontal cortex. Nat Neurosci (2012) 15(2):315-20. doi:10.1038/nn.3016

38. Sprague TC, Ester EF, Serences JT. Reconstructions of information in visual spatial working memory degrade with memory load. Curr Biol (2014) 24(18):2174-80. doi:10.1016/j.cub.2014.07.066
39. Mehta UM, Basavaraju R, Thirthalli J. Mirror neuron disinhibition may be linked with catatonic echo-phenomena: a single case TMS study. Brain Stimulat (2013) 6(4):705-7. doi:10.1016/j.brs.2012.12.002

40. Schulte-Rüther M, Markowitsch HJ, Fink GR, Piefke M. Mirror neuron and theory of mind mechanisms involved in face-to-face interactions: a functiona magnetic resonance imaging approach to empathy. J Cogn Neurosci (2007) 19(8):1354-72. doi:10.1162/jocn.2007.19.8.1354

41. Ene-Stroescu V, Nguyen T, Waiblinger BE. Excellent response to amantadine in a patient with bipolar disorder and catatonia. J Neuropsychiatry Clin Neurosci (2014) 26(1):E43. doi:10.1176/appi.neuropsych.13020038

42. Carroll BT, Goforth HW, Thomas C, Ahuja N, McDaniel WW, Kraus $\mathrm{MF}$, et al. Review of adjunctive glutamate antagonist therapy in the treatment of catatonic syndromes. J Neuropsychiatry Clin Neurosci (2007) 19(4):406-12. doi:10.1176/jnp.2007.19.4.406

43. Lee EM, Kang JK, Oh JS, Kim JS, Shin Y-W, Kim C-Y. 18F-fluorodeoxyglucose positron-emission tomography findings with anti-N-methyl-D-aspartate receptor encephalitis that showed variable degrees of catatonia: three cases report. J Epilepsy Res (2014) 4(2):69-73.

44. Yoshimura B, Yada Y, Horigome T, Kishi Y. Anti-N-methyl-D-aspartate receptor encephalitis presenting with intermittent catatonia. Psychosomatics (2014) 56(3):313-5. doi:10.1016/j.psym.2014.06.005

45. Duncan NW, Wiebking C, Northoff G. Associations of regional GABA and glutamate with intrinsic and extrinsic neural activity in humans - a review of multimodal imaging studies. Neurosci Biobehav Rev (2014) 47:36-52. doi:10.1016/j.neubiorev.2014.07.016

46. Eckstein M, Becker B, Scheele D, Scholz C, Preckel K, Schlaepfer TE, et al. Oxytocin facilitates the extinction of conditioned fear in humans. Biol Psychiatry (2014) 78(3):194-202. doi:10.1016/j. biopsych.2014.10.015

47. Kalk NJ, Lingford-Hughes AR. The clinical pharmacology of acamprosate. $\mathrm{Br}$ J Clin Pharmacol (2014) 77(2):315-23. doi:10.1111/bcp.12070

48. Narayanaswamy JC, Tibrewal P, Zutshi A, Srinivasaraju R, Math SB. Clinical predictors of response to treatment in catatonia. Gen Hosp Psychiatry (2012) 34(3):312-6. doi:10.1016/j.genhosppsych.2012.01.011

Conflict of Interest Statement: The authors declare that the research was conducted in the absence of any commercial or financial relationships that could be construed as a potential conflict of interest.

Copyright (c) 2015 Ellul and Choucha. This is an open-access article distributed under the terms of the Creative Commons Attribution License (CC BY). The use, distribution or reproduction in other forums is permitted, provided the original author(s) or licensor are credited and that the original publication in this journal is cited, in accordance with accepted academic practice. No use, distribution or reproduction is permitted which does not comply with these terms. 